\title{
Multi-Step Predictions for Adaptive Sampling using Proximal ADMM
}

\author{
Viet-Anh Le ${ }^{1}$, Linh Nguyen ${ }^{2}$ and Truong X. Nghiem ${ }^{1}$
}

\begin{abstract}
This paper presents a novel approach, using multistep predictions, to the adaptive sampling problem for efficient monitoring of environmental spatial phenomena in a resourceand obstacle-constrained mobile sensor network. We employ a Gaussian process (GP) to represent the spatial field of interest, which can then be used to predict the field at unmeasured locations. The adaptive sampling problem aims to drive the mobile sensors to optimally navigate the environment where the sensors adaptively take measurements of the spatial phenomena at each sampling step. To this end, an optimal sampling criterion based on conditional entropy is proposed, which aims to minimize the prediction uncertainty of the GP model. By predicting the measurements the mobile sensors potentially take in a finite horizon of multiple future sampling steps and exploiting the chain rule of the conditional entropy, a multi-step-ahead adaptive sampling optimization problem is formulated. The objective of the optimization problem is to find the optimal sampling paths for the mobile sensors in multiple sampling steps ahead. Compared with the single-step-ahead approach typically adopted in the literature, our approach provides better navigation, deployment and data collection with more informative sensor readings. However, this optimization problem is non-convex and highly complex. Even more challenging is that it is mixed-integer problem, due to collision avoidance constraints. We propose to employ the proximal alternating direction method of multipliers algorithm to efficiently solve this problem. More importantly, the solution obtained by the proposed algorithm is theoretically guaranteed to converge to a stationary value. The effectiveness of our proposed approach was extensively validated by simulation using a realworld dataset, which showed highly promising results.
\end{abstract}

Index Terms-Adaptive sampling, multi-step prediction, mobile robotic sensor networks, Gaussian processes, proximal ADMM, convergence analysis.

\section{INRODUCTION}

$\mathbf{T}$ HERE has been increasing demand for environmental monitoring in numerous applications such as monitoring water quality and air pollution [1], predicting disasters [2], controlling smart buildings [3], and target searching [4]. In such applications, the use of a mobile robotic sensor network (MRSN) has become increasingly popular due to several advantages, such as the ability to simultaneously collect data of various phenomena and at different locations, and the safety benefits for humans in dangerous environments such as in disasters. The data collected by an MRSN is usually incorporated with a machine learning (ML) model so that the MRSN is able to predict the field at any unobserved locations of interest. For

${ }^{1}$ V-A. Le and T. Nghiem are with the School of Informatics, Computing, and Cyber Systems, Northern Arizona University, Flagstaff, AZ 86011 USA $\{$ vl385, truong.nghiem\} @ nau.edu

${ }^{2}$ L. Nguyen is with the School of Engineering, Information Technology and Physical Sciences, Federation University Australia, Churchill, VIC 3842, Australia l.nguyen@ federation.edu.au example, Gaussian processes (GPs) [5] are frequently adopted in environmental monitoring applications [6]. A GP model can effectively learn a spatial phenomenon from a limited number of observations and then give an estimate of the spatial field along with the associated uncertainty at any location of interest. To optimize the operation of an MRSN, the mobile sensors are expected to take measurements at locations where the information gained by the sensor measurements is maximized so that the prediction uncertainty at all unobserved locations is minimized. This fundamental problem is widely known as adaptive sampling [7]. An efficient adaptive sampling strategy may reduce the numbers of deployed sensors and measurements needed for learning an accurate model of the field. Given resource constraints in the network, such as limited communication, computation, memory, and power capabilities, and constraints on robot mobility, such as collision avoidance and actuator limitation, formulating and solving the adaptive sampling problem are generally not straightforward. Research on the adaptive sampling problem for MRSNs has received increasing attention in recent years, of which comprehensive reviews can be found in [8], [9]. A brief summary of some recent studies related to our work will be given in Section II.

To the best of our knowledge, all existing methods for adaptive sampling in MRSNs employ single-step predictions. That is, by predicting the potential measurements the mobile robotic sensors can take in the next step only, the sampling strategy can decide where the robots should move to. In contrast, we propose in this work a novel approach for adaptive sampling using multi-step predictions. Unlike existing approaches which aim to find the most informative locations for the mobile sensors only at the next sampling step, in our approach, the network seeks the optimal sampling paths for the mobile sensors for a horizon of multiple future time steps. Our approach leads to better navigation and deployment of the mobile sensors and, as a result, better data collection with more informative measurements. Once the multi-step adaptive sampling problem is solved, the robots move to the first locations of their optimal sampling paths in the next time step to take new measurements. This procedure is repeated at every sampling time step in a receding horizon manner.

The adaptive sampling objective can be shown to be equivalent to maximization of the conditional entropy of the spatial field at the sampling locations of the robotic agents over the prediction horizon. This conditional entropy can be represented by the log determinant of the GP posterior covariance matrix at these locations. Moreover, to guarantee avoidance of inter-robot and robot-obstacle collisions at each time step of the horizon, we employ a scheme based on mixed- 
integer programming (MIP) [10]. The resulting optimization problem is non-convex and complex, and the log determinant of the GP posterior covariance matrix in the objective function may cause computational intractability. To effectively and efficiently solve this adaptive sampling optimization problem, similar to our previous work [11], we propose to use the proximal alternating direction method of multipliers (proximal ADMM) algorithm [12], [13]. More importantly, we formally prove that the proximal ADMM algorithm can obtain a stationary solution of the adaptive sampling problem if the covariance function of the GP model representing the spatial field is the squared exponential (SE) kernel. Extensive numerical simulations based on a real-world dataset were conducted to verify our proposed approach. The experimental results demonstrate that exploiting multi-step predictions for long-term planning can enhance the accuracy of the spatial field model.

In summary, our contributions in this paper are threefold:

1) A new adaptive sampling optimization problem for a resource-constrained MRSN is formulated, where the objective function is derived from multi-step predictions and collision avoidance is guaranteed by mixed integer constraints.

2) An efficient proximal ADMM based algorithm is proposed to effectively and efficiently solve the non-convex and complex constrained mixed-integer optimization problem for adaptive sampling.

3) A convergence analysis of the proposed algorithm is presented, which theoretically guarantees the convergence to a stationary solution when the SE kernel is used in the GP model.

The remainder of this paper is structured as follows. Related works are summarized in Section II. Section III introduces the proposed adaptive sampling approach for a MRSN to efficiently monitor a spatial field using multi-step predictions. Section IV presents an algorithm for solving the adaptive sampling optimization problem based on the proximal ADMM algorithm. Its convergence analysis is discussed in Section V. The effectiveness of the proposed approach is demonstrated in Section VI before conclusions are drawn in Section VII.

\section{RELATED WORKS}

The adaptive sampling problem in an MRSN for environment monitoring has been widely studied in the literature. For instance, the authors of [14] presented a method to partition an MRSN into several small groups, in which each group is responsible for finding its own optimal sampling locations. Likewise, Tiwari et al., [15] considered a decentralized multirobot system where each robot is allocated a local sensing zone for monitoring. By exploiting the Voronoi concept, the authors of [16] proposed a method that allows each sensing agent to conduct its adaptive sampling tasks within its dynamic cell area. Some studies [17], [18] used a flocking control approach to form a network of mobile sensors that track a virtual leader, leading to a path planning problem for each agent.

In the majority of existing works, the mobile sensors jointly find their optimal sampling locations in the entire region of interest. In such cases, a prediction metric is usually used to formulate the optimization problem. For example, the authors of [19] proposed to determine the next sampling locations of the sensing agents by maximizing their predictive variances. In [20], the maximum a posteriori estimation technique was employed to design an adaptive sampling strategy that aims to minimize predictive variances. In a similar fashion, an adaptive sampling problem was formulated in [21] where the cost function is the average of the predictive variances over a set of pre-specified target points. Xu et al., [22] later designed a strategy to minimize both prediction errors and uncertainty in hyperparameters simultaneously. Nevertheless, the work [23], at a different angle, formalizes an active sensing criterion that trades off between gathering the most informative observations and predicting the phenomenon given the current but possibly inaccurate estimate of the correlation structure. Considering balance between exploration and exploitation in a Bayesian optimization problem, Marchant et al., in [24] exploited traveled distances of mobile robots to derive a sampling strategy. The authors in [25] derived a constrained dynamical optimization problem with two objectives that correspondingly locate peaks of environmental data and guarantee robot coordination. A reinforcement learning based method was proposed in [26] to minimize the log volume of a confidence ellipsoid representing an estimation error. In [27], both the mean value for exploitation and the variance estimate for exploration were maximized given collision avoidance constraints to find the next sampling locations. Furthermore, the multi-robot coverage control has been taken into account simultaneously with the sampling design in [28], [29]. Other metrics having been utilized in adaptive sampling are based on information criteria, including Fisher information matrix, entropy, and mutual information [30]-[32]. In [33], the upper confidence bound of a GP was adopted in an optimization objective and the cross-entropy method was used to optimize the sampling paths. In [34], [35], a Gaussian Markov random field was exploited to represent a spatial field on an irregular discrete lattice and the adaptive sampling strategies were designed based on mutual information and conditional entropy, respectively.

The adaptive sampling optimization problems using metrics of joint predictions for the entire sensor networks are generally non-convex with complex objective functions. Therefore, methods for solving the problems efficiently have been investigated in some recent studies. For example, in [36], [37], it was shown that a grid-based greedy method can be employed to solve the adaptive sampling problems where submodular properties are exploited to prove the solution bounds. However, we have demonstrated that solutions of the adaptive sampling problem can be improved by solving it in a continuous domain. More specifically, in [11], we proposed to solve the problem by the proximal ADMM algorithm, that uses proximal operators to deal with the complex adaptive sampling objective function and the constraints on robot mobility separately. The results in [11] showed that the proximal ADMM method can outperform the commonly used grid-based greedy algorithm. In [38], we extended the algorithm to handle non-holonomic dynamics of the robots in an MRSN using distributed computation, 
which has not been considered in previous works, and obtained highly promising results.

Nevertheless, in all the previous works, the mobile sensors decide where to move to next based on predictions at only the next sampling step. This single-step prediction strategy could be short-sighted and result in sub-optimal adaptive sampling since it does not fully exploit the prior information available. In this paper, we propose a novel multi-step prediction approach for adaptive sampling, that looks multiple steps into the future to seek the optimal sampling path.

\section{Multi-Step PREDiCTION APPROACH FOR AdAPTIVE SAMPLING OPTIMIZATION}

This section introduces a new approach based on multi-step predictions for the adaptive sampling problem of a MRSN in environmental monitoring. At each sampling instant, it seeks the most informative sampling paths of the mobile sensors in a prediction horizon of $H$ time steps, which are then employed to decide where the sensors should move to in the next sampling step.

\section{A. Environment Modeling in MRSNs with Gaussian Processes}

Consider a network of $M$ mobile robots, where each robot is equipped with a sensor to take measurements of a spatial field. We define $\mathcal{V}=\{1, \ldots, M\}$ as the set of robot indices. We denote $\mathbf{s}_{i, t}=\left[s_{x, i, t}, s_{y, i, t}\right]^{T}$ as the location vector of robot $i$ at time step $t$ and assume that the locations of the robots are in a compact $2 \mathrm{D}$ Euclidean space of interest $\mathcal{Q} \subset \mathbb{R}^{2}$, i.e., $\mathbf{s}_{i, t} \in \mathcal{Q}$ for all $i=1, \ldots, M$ and all $t \geq 0$. Let $\mathbf{s}_{t}=\left[\mathbf{s}_{i, t}\right]_{i=1, \ldots, M} \in$ $\mathbb{R}^{2 \times M}$ denote the matrix of all robotic sensor locations at time step $t$, and $\mathbf{s}_{t_{1}: t_{2}}=\left[\mathbf{s}_{k}\right]_{k=t_{1}, \ldots, t_{2}} \in \mathbb{R}^{2 \times\left(t_{2}-t_{1}+1\right) M}$ denote the collective matrix of robot locations from time step $t_{1}$ to time step $t_{2}$. We restrict the distance travelled by each robot at two consecutive sampling steps as

$$
\Delta \mathbf{s}_{\min } \leq \mathbf{s}_{i, t}-\mathbf{s}_{i, t-1} \leq \Delta \mathbf{s}_{\max }
$$

At each time step, the mobile sensor $i$ takes a noisy measurement $y_{i, t}$ of the spatial field of interest at its current location, which is modeled as

$$
y_{i, t}=h\left(\mathbf{s}_{i, t}\right)+w_{i},
$$

where $h: \mathbb{R}^{2} \rightarrow \mathbb{R}$ is a latent function representing the spatial phenomenon and $w_{i}$ is an independent and identically distributed zero-mean Gaussian noise. All the sensor measurements at time step $t$ are denoted by $\mathbf{y}_{t}=\left[y_{i, t}\right]_{i=1, \ldots, M} \in \mathbb{R}^{M}$ and the collective measurements from time step $t_{1}$ to time step $t_{2}$ are denoted by $\mathbf{y}_{t_{1}: t_{2}}=\left[\mathbf{y}_{k}\right]_{k=t_{1}, \ldots, t_{2}} \in \mathbb{R}^{\left(t_{2}-t_{1}+1\right) M}$.

In environmental monitoring, a GP is commonly employed to learn a model of the latent spatial field from the collective sensor measurements. A GP derives a spatial relationship between sampled data by using a kernel (or covariance function) and performs Bayesian inference for predictions at unobserved locations. At time $t$, let $\mathcal{G}_{t}$ denote the GP model trained on the data set $\mathcal{D}_{t}=\left(\mathbf{s}_{0: t}, \mathbf{y}_{0: t}\right)$, i.e., the locations and measurements taken from all mobile sensors up to time $t$. Statistically, $\mathcal{G}_{t}$ is fully specified by a mean function $m\left(\mathbf{s}^{\prime} ; \boldsymbol{\theta}_{t}\right)$ and a kernel $\kappa\left(\mathbf{s}^{\prime}, \mathbf{s}^{\prime \prime} ; \boldsymbol{\theta}_{t}\right)$ that are parameterized by the hyperparameters $\boldsymbol{\theta}_{t}$.
The hyperparameters $\boldsymbol{\theta}_{t}$ can be obtained by maximizing the likelihood [5]. At time $t+1$, the joint predictions at $\mathbf{s}_{t+1}$ of $\mathcal{G}_{t}$ is a random vector $\hat{\mathbf{y}}_{t+1} \sim \mathcal{N}\left(\boldsymbol{\mu}_{\hat{\mathbf{y}}_{t+1} \mid \mathbf{y}_{0: t}}, \boldsymbol{\Sigma}_{\hat{\mathbf{y}}_{t+1} \mid \mathbf{y}_{0: t}}\right)$, in which the posterior mean vector $\boldsymbol{\mu}_{\hat{\mathbf{y}}_{t+1} \mid \mathbf{y}_{0: t}}$ and the posterior covariance matrix $\boldsymbol{\Sigma}_{\hat{\mathbf{y}}_{t+1} \mid \mathbf{y}_{0: t}}$ are computed as

$$
\begin{aligned}
& \boldsymbol{\mu}_{\hat{\mathbf{y}}_{t+1} \mid \mathbf{y}_{0: t}}=\mathbf{m}_{t+1}+\mathbf{K}_{t+1}\left(\mathbf{K}+\sigma_{n}^{2} \mathbb{I}\right)^{-1}\left(\mathbf{y}_{0: t}-\mathbf{m}_{0: t}\right) \\
& \boldsymbol{\Sigma}_{\hat{\mathbf{y}}_{t+1} \mid \mathbf{y}_{0: t}}=\mathbf{K}_{t+1, t+1}-\mathbf{K}_{t+1}\left(\mathbf{K}+\sigma_{n}^{2} \mathbb{I}\right)^{-1} \mathbf{K}_{t+1}^{T}
\end{aligned}
$$

Here, $\sigma_{n}^{2}>0$ is the Gaussian noise variance and $\mathbb{I}$ is an identity matrix of appropriate dimensions. Vectors $\mathbf{m}_{t+1}$ and $\mathbf{m}_{0: t}$ are the prior mean vectors at $\mathbf{s}_{t+1}$ and $\mathbf{s}_{0: t}$, respectively, whose element $m_{i}$ is computed by $m_{i}=m\left(\mathbf{s}^{(i)}\right)$, where $\mathbf{s}^{(i)}$ denotes the vector of the $i$-th column in the corresponding input matrix. $\mathbf{K}_{t+1, t+1} \in \mathbb{R}^{M \times M}$ is the covariance matrix at $\mathbf{s}_{t+1}$ and $\mathbf{K}_{t+1} \in \mathbb{R}^{M \times M(t+1)}$ is the cross-covariance matrix between $\mathbf{s}_{t+1}$ and $\mathbf{s}_{0: t} . \mathbf{K} \in \mathbb{R}^{M(t+1) \times M(t+1)}$ is the covariance matrix at $\mathbf{s}_{0: t}$. These matrices are defined such that the $(i, j)$ element of each is $K_{i j}=\kappa\left(\mathbf{s}^{(i)}, \mathbf{s}^{(j)}\right)$.

\section{B. Adaptive Sampling with Multi-Step Predictions}

Let $t$ be the current time step and $H \geq 1$ be a prediction horizon length. Let us denote $\mathcal{I}_{t}=\{t+1, \ldots, t+H\}$ as the set of all time steps in the prediction horizon. The past sampling locations $\mathbf{s}_{0: t}$ and observations $\mathbf{y}_{0: t}$ are known, on which the GP model $\mathcal{G}_{t}$ was learned.

For any $k \in \mathcal{I}_{t}$, let $\mathbf{s}_{t+1: k-1}$ be the future locations of the robots up to time $k-1$, at which the predictive observations as calculated by $\mathcal{G}_{t}$ are $\hat{\mathbf{y}}_{t+1: k-1}$. Note that $\hat{\mathbf{y}}_{t+1: k-1}$ are predicted by $\mathcal{G}_{t}$ given the available information at time $t$, not the actual observations. To predict the observations at time $k$, we use the data set $\hat{\mathcal{D}}_{k-1}=\left(\mathbf{s}_{0: k-1}, \hat{\mathbf{y}}_{0: k-1}\right)$ available at time $k-1$, where $\mathbf{s}_{0: k-1}=\left[\mathbf{s}_{0: t}, \mathbf{s}_{t+1: k-1}\right]$ and $\hat{\mathbf{y}}_{0: k-1}=\left[\mathbf{y}_{0: t}, \hat{\mathbf{y}}_{t+1: k-1}\right]$. From (3), the posterior covariance matrix for the predictions $\hat{\mathbf{y}}_{k}$ at the sensor locations $\mathbf{s}_{k}$ is given by

$$
\boldsymbol{\Sigma}_{\hat{\mathbf{y}}_{k} \mid \hat{\mathbf{y}}_{0: k-1}}=\mathbf{K}_{k, k}-\mathbf{K}_{k}\left(\mathbf{K}+\sigma_{n}^{2} \mathbb{I}\right)^{-1} \mathbf{K}_{k}^{T}
$$

It has been shown that the next optimal locations for the mobile sensors can be obtained by maximizing the conditional entropy of the spatial field at the next sampling locations given the current GP model [36]. The conditional entropy is computed by the log determinant of the GP predictive covariance matrix. Thus, in a single-step prediction strategy, the optimization problem for adaptive sampling is formulated as $\mathbf{s}_{t+1}^{*}=\operatorname{argmin}_{s_{t+1} \in \mathcal{Q}}-\log \operatorname{det} \boldsymbol{\Sigma}_{\hat{\mathbf{y}}_{t+1} \mid \mathbf{y}_{0: t}}\left(\mathbf{s}_{t+1}\right)$.

We will extend the above result to account for multi-step predictions. Using the same argument, the optimal sequence of locations for the horizon $\mathcal{I}_{t}$ can be found by maximizing the conditional entropy of the spatial field between the present (at time $t$ ) and the future after $H$ time steps, which is given by $\sum_{k \in \mathcal{I}_{t}}-\log \operatorname{det} \boldsymbol{\Sigma}_{\hat{\mathbf{y}}_{k} \mid \hat{\mathbf{y}}_{0: k-1}}\left(\mathbf{s}_{k}\right)$. In other words, the optimal locations $\mathbf{s}_{\mathcal{I}_{t}}^{*}$ can be found by solving the following optimization problem

$$
\mathbf{s}_{\mathcal{I}_{t}}^{*}=\underset{s_{\mathcal{I}_{t}} \in \mathcal{Q}}{\operatorname{argmin}} \sum_{k \in \mathcal{I}_{t}}-\log \operatorname{det} \boldsymbol{\Sigma}_{\hat{\mathbf{y}}_{k} \mid \hat{\mathbf{y}}_{0: k-1}}\left(\mathbf{s}_{k}\right),
$$




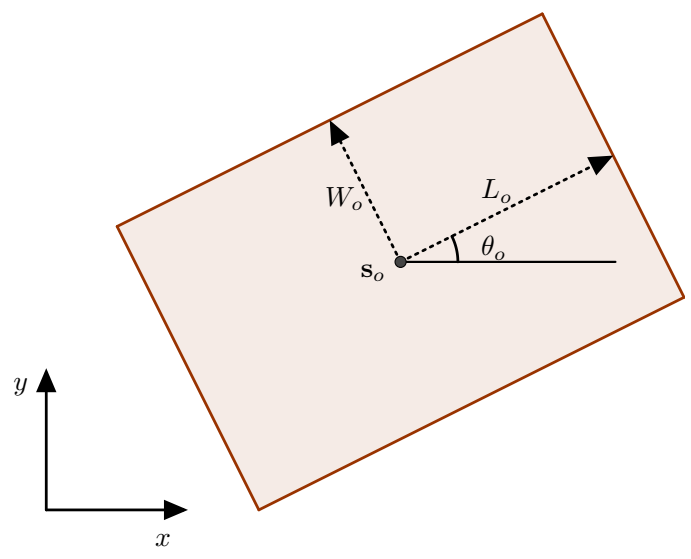

Fig. 1: Illustration of a rectangular obstacle.

where $\boldsymbol{\Sigma}_{\hat{\mathbf{y}}_{k} \mid \hat{\mathbf{y}}_{0: k-1}}\left(\mathbf{s}_{k}\right)$ is given by (4). Using the chain rule for the conditional entropy [39], the objective function of (5) can be simplified as

$$
\begin{aligned}
& \sum_{k \in \mathcal{I}_{t}}-\log \operatorname{det} \boldsymbol{\Sigma}_{\hat{\mathbf{y}}_{k} \mid \hat{\mathbf{y}}_{0: k-1}}\left(\mathbf{s}_{k}\right) \\
= & -\log \operatorname{det} \boldsymbol{\Sigma}_{\hat{\mathbf{y}}_{\mathcal{I}_{t}} \mid \mathbf{y}_{0: t}}\left(\mathbf{s}_{\mathcal{I}_{t}}\right) .
\end{aligned}
$$

The covariance matrix $\boldsymbol{\Sigma}_{\hat{\mathbf{y}}_{\mathcal{I}_{t}} \mid \mathbf{y}_{0: t}}\left(\mathbf{s}_{\mathcal{I}_{t}}\right)$ is computed by

$$
\boldsymbol{\Sigma}_{\hat{\mathbf{y}}_{\mathcal{I}_{t}} \mid \mathbf{y}_{0: t}}\left(\mathbf{s}_{\mathcal{I}_{t}}\right)=\mathbf{K}_{\mathcal{I}_{t}, \mathcal{I}_{t}}-\mathbf{K}_{\mathcal{I}_{t}}\left(\mathbf{K}+\sigma_{n}^{2} \mathbb{I}\right)^{-1} \mathbf{K}_{\mathcal{I}_{t}}^{T},
$$

where $\mathbf{K}_{\mathcal{I}_{t}, \mathcal{I}_{t}} \in \mathbb{R}^{M H \times M H}$ is the covariance matrix at $\mathbf{s}_{\mathcal{I}_{t}}$ and $\mathbf{K}_{\mathcal{I}_{t}} \in \mathbb{R}^{M H \times M(t+1)}$ is the cross-covariance matrix between $\mathbf{s}_{\mathcal{I}_{t}}$ and $\mathbf{s}_{0: t}$.

For brevity, we denote the matrix $\boldsymbol{\Sigma}_{\hat{\mathbf{y}}_{\mathcal{I}_{t}} \mid \mathbf{y}_{0: t}}\left(\mathbf{s}_{\mathcal{I}_{t}}\right)$ by $\boldsymbol{\Sigma}(\mathbf{s})$ henceforth, where $\mathbf{s} \in \mathbb{R}^{2 M H}$ is a vector that includes all variables in the matrix $\mathbf{s}_{\mathcal{I}_{t}}$. To avoid numerical issues in the implementation due to possible singularity of the covariance matrix (see Lemma 1 below) and to guarantee convergence of the optimization algorithm, a small term $\epsilon^{2} \mathbb{I}$ is added to the covariance matrix (7), where $\epsilon^{2}$ is a small positive constant. Therefore, our multi-step predictions strategy for adaptive sampling solves the following problem

$$
\mathbf{s}^{*}=\underset{s \in \mathcal{Q}}{\operatorname{argmin}} \mathcal{H}(\mathbf{s})
$$

where $\mathcal{H}(\mathbf{s})=-\log \operatorname{det}\left(\boldsymbol{\Sigma}(\mathbf{s})+\epsilon^{2} \mathbb{I}\right)$.

Lemma 1: The matrices $\boldsymbol{\Sigma}(\mathbf{s})+\epsilon^{2} \mathbb{I}$ and $\mathbf{K}+\sigma_{n}^{2} \mathbb{I}$ are invertible and their inverse matrices are bounded.

Proof: Since $\mathbf{K}$ is positive semi-definite (PSD) [5], for any eigenvalue $\lambda_{i}$ of $\mathbf{K}$ we have $\lambda_{i} \geq 0$. The eigenvalues of $\mathbf{K}+\sigma_{n}^{2} \mathbb{I}$ are $\lambda_{i}+\sigma_{n}^{2}$, which are lower-bounded by the positive constant $\sigma_{n}^{2}$. Therefore, its inverse matrix exists and is bounded.

Similarly for $\boldsymbol{\Sigma}(\mathbf{s})$, its non-singularity and the boundedness of its inverse can be established.

\section{Collision Avoidance}

To avoid robot-robot and robot-obstacle collisions, we employ the MIP scheme proposed in [10]. A collision between two agents $i$ and $j$ at every time step can be avoided by the following constraint, where the time-step subscript $t$ is omitted for brevity and $d_{i j}$ is a safety distance between the agents,

$$
\left|s_{x, i}-s_{x, j}\right| \geq d_{i j} \quad \text { OR } \quad\left|s_{y, i}-s_{y, j}\right| \geq d_{i j} .
$$

This constraint can be represented equivalently by the following conjunction

$$
\begin{gathered}
s_{x, i}-s_{x, j} \geq d_{i j}-M\left(c_{1, i j}+c_{2, i j}\right) \\
\text { AND } s_{x, i}-s_{x, j} \geq d_{i j}-M\left(1-c_{1, i j}+c_{2, i j}\right) \\
\text { AND } s_{y, i}-s_{y, j} \geq d_{i j}-M\left(1+c_{1, i j}-c_{2, i j}\right) \\
\text { AND } s_{y, i}-s_{y, j} \geq d_{i j}-M\left(2-c_{1, i j}-c_{2, i j}\right),
\end{gathered}
$$

where $c_{1, i j}, c_{2, i j} \in\{0,1\}$ are binary variables, and $M$ is a large positive constant.

For obstacle avoidance, we assume that the obstacles are stationary, known in advance, and represented by rectangles as illustrated in Figure 1. Nonetheless, our method can be extended to any polyhedral obstacles. Define $\left(s_{x, o}, s_{y, o}\right), \theta_{o}$, $2 L_{o}$, and $2 W_{o}$ as the center location, the rotation angle, the length and the width of obstacle $o$, respectively. Each robot $i$ must stay outside the area of obstacle $o$, which is expressed by the following disjunctive statements

$$
\begin{gathered}
\cos \theta_{o}\left(s_{x, i}-s_{x, o}\right)+\sin \theta_{o}\left(s_{y, i}-s_{y, o}\right) \geq L_{o} \\
\text { OR }-\cos \theta_{o}\left(s_{x, i}-s_{x, o}\right)-\sin \theta_{o}\left(s_{y, i}-s_{y, o}\right) \geq L_{o} \\
\text { OR } \sin \theta_{o}\left(s_{x, i}-s_{x, o}\right)-\cos \theta_{o}\left(s_{y, i}-s_{y, o}\right) \geq W_{o} \\
\text { OR }-\sin \theta_{o}\left(s_{x, i}-s_{x, o}\right)+\cos \theta_{o}\left(s_{y, i}-s_{y, o}\right) \geq W_{o} .
\end{gathered}
$$

This condition is equivalent to the following constraints

$$
\begin{array}{r}
\cos \theta_{o}\left(s_{x, i}-s_{x, o}\right)+\sin \theta_{o}\left(s_{y, i}-s_{y, o}\right) \\
\geq L_{o}-M\left(c_{1, i o}+c_{2, i o}\right) \\
\text { AND }-\cos \theta_{o}\left(s_{x, i}-s_{x, o}\right)-\sin \theta_{o}\left(s_{y, i}-s_{y, o}\right) \\
\geq L_{o}-M\left(1+c_{1, i o}-c_{2, i o}\right) \\
\text { AND } \sin \theta_{o}\left(s_{x, i}-s_{x, o}\right)-\cos \theta_{o}\left(s_{y, i}-s_{y, o}\right) \\
\geq W_{o}-M\left(2-c_{1, i o}-c_{2, i o}\right) \\
\text { AND }-\sin \theta_{o}\left(s_{x, i}-s_{x, o}\right)+\cos \theta_{o}\left(s_{y, i}-s_{y, o}\right) \\
\geq W_{o}-M\left(1-c_{1, i o}+c_{2, i o}\right),
\end{array}
$$

where $c_{1, i o}, c_{2, i o} \in\{0,1\}$.

\section{Adaptive Sampling Optimization Formulation}

Given the adaptive sampling objective (6), the robot mobility constraints (1), and the MIP collision avoidance constraints, the adaptive sampling problem using multi-step predictions can be formulated as

$$
\begin{aligned}
& \underset{\mathbf{s}, \mathbf{c}}{\operatorname{minimize}} \mathcal{H}(\mathbf{s}) \\
& \text { subject to } \\
& \qquad \mathbf{s}_{i, k} \in \mathcal{Q}, \forall i \in \mathcal{V}, \forall k \in \mathcal{I}_{t} \\
& \qquad \Delta \mathbf{s}_{\min } \leq \mathbf{s}_{i, k}-\mathbf{s}_{i, k-1} \leq \Delta \mathbf{s}_{\max }, \forall i \in \mathcal{V}, \forall k \in \mathcal{I}_{t} \\
& \quad \text { (9) and (10), } \forall i \neq j \in \mathcal{V}, \forall k \in \mathcal{I}_{t},
\end{aligned}
$$

where $\mathbf{c}$ denotes the concatenated vector of all binary variables over the horizon. To facilitate the convergence analysis, we redefine $\mathbf{c}$ as a real vector and constrain it in a non-convex cone 
where only two elements $\{0,1\}$ exist. Moreover, we represent the non-convex cone constraint and all the constraints in (11) by $\mathbf{s}, \mathbf{c} \in \mathcal{C}$, leading to the following simplified version of (11)

$$
\begin{array}{cl}
\underset{\mathbf{s}, \mathbf{c}}{\operatorname{minimize}} & \mathcal{H}(\mathbf{s}) \\
\text { subject to } & \{\mathbf{s}, \mathbf{c}\} \in \mathcal{C} .
\end{array}
$$

It can be seen that (12) is a mixed integer nonlinear program, where the complexity of the objective function involving the log determinant of a $M H \times M H$ covariance matrix can lead to computational intractability [11]. To address this challenge, we propose to apply the proximal ADMM algorithm [12], [13] to solve this problem efficiently.

\section{Proximal ADMM for Multi-Step Predictions BASED ADAPTIVE SAMPLING}

In our previous work [11], the proximal ADMM algorithm was applied to solve the adaptive sampling problem with single-step predictions. In this section, we will extend the algorithm to efficiently solve the multi-step predictions based adaptive sampling problem.

Let $\mathbf{x}=\left[\mathbf{s}^{T}, \mathbf{c}^{T}\right]^{T}$ be the concatenated vector of all optimization variables. We then define a matrix $\mathbf{F}$ that extracts $\mathbf{s}$ from $\mathbf{x}$, i.e., $\mathbf{s}=\mathbf{F x}$. Hence, the objective function in (12) can be rewritten as $f(\mathbf{x})=\mathcal{H}(\mathbf{F} \mathbf{x})$. Let $I_{\mathcal{C}}(\cdot)$ denote the indicator function of $\mathcal{C}$. Then the problem (12) can be written in the consensus form as

$$
\begin{array}{ll}
\underset{\mathbf{x}, \mathbf{z}}{\operatorname{minimize}} & f(\mathbf{x})+I_{\mathcal{C}}(\mathbf{z}) \\
\text { subject to } & \mathbf{x}=\mathbf{z},
\end{array}
$$

where $\mathbf{z}$ is a copy of $\mathbf{x}$. The augmented Lagrangian function of the problem (13) is defined by

$$
\mathcal{L}(\mathbf{x}, \mathbf{z}, \mathbf{u})=f(\mathbf{x})+I_{\mathcal{C}}(\mathbf{z})+\mathbf{u}^{T}(\mathbf{x}-\mathbf{z})+\frac{\rho}{2}\|\mathbf{x}-\mathbf{z}\|_{2}^{2},
$$

where $\mathbf{u}$ is a vector of the associated dual variables and $\rho$ is a regularization parameter. The non-convex ADMM algorithm in [40] can be applied to solve the optimization problem (13) in the following steps at each iteration.

$$
\begin{gathered}
\mathbf{z}^{(k+1)}=\underset{\mathbf{z} \in \mathcal{C}}{\operatorname{argmin}}\left\|\mathbf{z}-\left(\mathbf{x}^{k}+(1 / \rho) \mathbf{u}^{(k)}\right)\right\|_{2}^{2} \\
\mathbf{x}^{(k+1)}=\underset{\mathbf{x}}{\operatorname{argmin}} f(\mathbf{x})+\left(\mathbf{u}^{(k)}\right)^{T}\left(\mathbf{x}-\mathbf{z}^{(k+1)}\right) \\
+\frac{\rho}{2}\left\|\mathbf{x}-\mathbf{z}^{(k+1)}\right\|_{2}^{2} \\
\mathbf{u}^{(k+1)}=\mathbf{u}^{(k)}+\rho\left(\mathbf{z}^{(k+1)}-\mathbf{s}^{(k+1)}\right) .
\end{gathered}
$$

However, it can be seen that exactly solving the $\mathrm{x}$ minimization step (15b), which involves the log determinant of the predictive covariance matrix of the GP, is computationally intractable. To deal with the complexity of $f(\cdot)$, the proximal ADMM algorithm developed in [12], [13] exploits its first-
Algorithm 1 Proximal ADMM algorithm for multi-step predictions based adaptive sampling problem

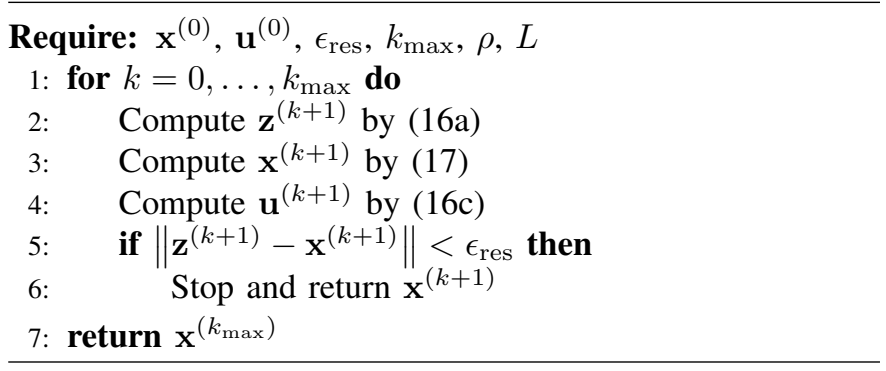

order approximation to obtain tractable computation steps for solving (13) as follows

$$
\begin{aligned}
\mathbf{z}^{(k+1)} & =\underset{\mathbf{z} \in \mathcal{C}}{\operatorname{argmin}}\left\|\mathbf{z}-\left(\mathbf{x}^{k}+(1 / \rho) \mathbf{u}^{(k)}\right)\right\|_{2}^{2} \\
\mathbf{x}^{(k+1)} & =\underset{\mathbf{z}}{\operatorname{argmin}} \nabla^{T} f\left(\mathbf{z}^{(k+1)}\right)\left(\mathbf{x}-\mathbf{z}^{(k+1)}\right) \\
& +\left(\mathbf{u}^{(k)}\right)^{T}\left(\mathbf{x}-\mathbf{z}^{(k+1)}\right)+\frac{\rho+L}{2}\left\|\mathbf{x}-\mathbf{z}^{(k+1)}\right\|_{2}^{2} \\
\mathbf{u}^{(k+1)} & =\mathbf{u}^{(k)}+\rho\left(\mathbf{z}^{(k+1)}-\mathbf{s}^{(k+1)}\right),
\end{aligned}
$$

where $L$ is the Lipschitz constant of $\nabla f(\cdot)$. It is important to note that the $z$-minimization step (16a) is a convex mixedinteger quadratic program (MIQP), which can be solved efficiently by any MIQP solver, and the $x$-minimization step (16b) is an unconstrained convex quadratic minimization which has an analytical optimal solution given by

$$
\mathbf{x}^{(k+1)}=\mathbf{z}^{(k+1)}-\frac{1}{(\rho+L)}\left(\nabla f\left(\mathbf{z}^{(k+1)}\right)+\mathbf{u}^{(k)}\right) .
$$

The term $\nabla f\left(\mathbf{z}^{(k+1)}\right)$ in (17) is computed as follows. The gradient of $f(\cdot)$ with respect to $\mathbf{c}$ is vanished, while the derivative of $f(\cdot)$ with respect to each element $s_{j}$ of $\mathbf{s}$ is

$$
\frac{\partial f(\mathbf{x})}{\partial s_{j}}=\frac{\partial \mathcal{H}(\mathbf{s})}{\partial s_{j}}=-\operatorname{tr}\left(\left(\boldsymbol{\Sigma}(\mathbf{s})+\epsilon^{2} \mathbb{I}\right) \frac{\partial \boldsymbol{\Sigma}(\mathbf{s})}{\partial s_{j}}\right),
$$

where from (7) we have

$$
\frac{\partial \boldsymbol{\Sigma}(\mathbf{s})}{\partial s_{j}}=\frac{\partial \mathbf{K}_{\mathcal{I}_{t}, \mathcal{I}_{t}}}{\partial s_{j}}-2 \mathbf{K}_{\mathcal{I}_{t}}\left(\mathbf{K}+\sigma_{n}^{2} \mathbb{I}\right)^{-1}\left(\frac{\partial \mathbf{K}_{\mathcal{I}_{t}}}{\partial s_{j}}\right)^{T} .
$$

The steps of the proximal ADMM algorithm for solving the multi-step predictions based adaptive sampling problem are summarized in Algorithm 1. The algorithm is terminated when the norm of the dual errors $\left\|\mathbf{z}^{(k+1)}-\mathbf{x}^{(k+1)}\right\|$ is less than or equal to a pre-defined threshold $\epsilon_{\text {res }}$ or a maximum number of iterations $k_{\max }$ is reached. Note that at each iteration the proximal ADMM algorithm requires only one MIQP optimization problem in step (16a), which is more tractable than directly solving the original mixed-integer nonlinear programming problem (12).

\section{Convergence Analysis}

This section presents a convergence analysis of the proposed algorithm. We will show that if the widely-used squared exponential (SE) kernel is employed for the GP then the algorithm can converge to a stationary solution. 
Assumption 1: The SE kernel is used for the GP model $\mathcal{G}_{t}$. The SE kernel is given by

$$
\kappa\left(\mathbf{s}^{\prime}, \mathbf{s}^{\prime \prime}\right)=\sigma_{f}^{2} \exp \left(-\frac{1}{2}\left(\mathbf{s}^{\prime}-\mathbf{s}^{\prime \prime}\right)^{T} \boldsymbol{\Lambda}^{-1}\left(\mathbf{s}^{\prime}-\mathbf{s}^{\prime \prime}\right)\right),
$$

where $\boldsymbol{\Lambda}=\operatorname{diag}\left(\lambda_{1}^{2}, \lambda_{2}^{2}\right)$ is a diagonal matrix of the lengthscales and $\sigma_{f}^{2}$ is the variance of the kernel. It is important to remark that the SE kernel is assumed in this section to make explicit the mathematical derivations and the convergence analysis. Nonetheless, our approach and algorithm as presented in Sections III and IV are applicable to any kernels. The convergence analyses for other kernels will be investigated in future work.

We now derive some properties related to the SE kernel.

Lemma 2: Considering the SE kernel (20) for any pair of location vectors $\mathbf{s}^{\prime}=\left[s_{x}^{\prime}, s_{y}^{\prime}\right]^{T}$ and $\mathbf{s}^{\prime \prime}=\left[s_{x}^{\prime \prime}, s_{y}^{\prime \prime}\right]^{T}$, the following functions are bounded

1) $\kappa\left(\mathbf{s}^{\prime}, \mathbf{s}^{\prime \prime}\right)$.

2) $\partial \kappa\left(\mathbf{s}^{\prime}, \mathbf{s}^{\prime \prime}\right) / \partial s_{j}^{\prime}$ and $\partial \kappa\left(\mathbf{s}^{\prime}, \mathbf{s}^{\prime \prime}\right) / \partial s_{j}^{\prime \prime}$, for $j=x, y$.

3) $\partial^{2} \kappa\left(\mathbf{s}^{\prime}, \mathbf{s}^{\prime \prime}\right) / \partial s_{i}^{\prime} \partial s_{j}^{\prime}$ and $\partial^{2} \kappa\left(\mathbf{s}^{\prime}, \mathbf{s}^{\prime \prime}\right) / \partial s_{i}^{\prime \prime} \partial s_{j}^{\prime \prime}$, for $i=$ $x, y$ and $j=x, y$.

4) $\partial^{2} \kappa\left(\mathbf{s}^{\prime}, \mathbf{s}^{\prime \prime}\right) / \partial s_{i}^{\prime} \partial s_{j}^{\prime \prime}$ and $\partial^{2} \kappa\left(\mathbf{s}^{\prime}, \mathbf{s}^{\prime \prime}\right) / \partial s_{i}^{\prime \prime} \partial s_{j}^{\prime}$, for $i=$ $x, y$ and $j=x, y$.

Proof: We first present explicit forms of the functions in parts 2, 3 and 4 of Lemma 2. For part 2,

$$
\frac{\partial \kappa\left(\mathbf{s}^{\prime}, \mathbf{s}^{\prime \prime}\right)}{\partial s_{j}^{\prime}}=-\frac{1}{\lambda_{j}^{2}}\left(s_{j}^{\prime}-s_{j}^{\prime \prime}\right) \kappa\left(\mathbf{s}^{\prime}, \mathbf{s}^{\prime \prime}\right) .
$$

For part 3,

$$
\frac{\partial^{2} \kappa\left(\mathbf{s}^{\prime}, \mathbf{s}^{\prime \prime}\right)}{\partial s_{j}^{\prime} \partial s_{j}^{\prime}}= \begin{cases}-\frac{1}{\lambda_{j}^{2}}\left(1-\frac{\left(s_{j}^{\prime}-s_{j}^{\prime \prime}\right)^{2}}{\lambda_{j}^{2}}\right) \kappa\left(\mathbf{s}^{\prime}, \mathbf{s}^{\prime \prime}\right) & \text { if } i=j \\ \frac{1}{\lambda_{i}^{2} \lambda_{j}^{2}}\left(s_{i}^{\prime}-s_{i}^{\prime \prime}\right)\left(s_{j}^{\prime}-s_{j}^{\prime \prime}\right) \kappa\left(\mathbf{s}^{\prime}, \mathbf{s}^{\prime \prime}\right) & \text { if } i \neq j .\end{cases}
$$

For part 4,

$$
\frac{\partial^{2} \kappa\left(\mathbf{s}^{\prime}, \mathbf{s}^{\prime \prime}\right)}{\partial s_{i}^{\prime} \partial s_{j}^{\prime \prime}}= \begin{cases}\frac{1}{\lambda_{j}^{2}}\left(1-\frac{\left(s_{j}^{\prime}-s_{j}^{\prime \prime}\right)^{2}}{\lambda_{j}^{2}}\right) \kappa\left(\mathbf{s}^{\prime}, \mathbf{s}^{\prime \prime}\right) & \text { if } i=j \\ -\frac{1}{\lambda_{i}^{2} \lambda_{j}^{2}}\left(s_{i}^{\prime}-s_{i}^{\prime \prime}\right)\left(s_{j}^{\prime}-s_{j}^{\prime \prime}\right) \kappa\left(\mathbf{s}^{\prime}, \mathbf{s}^{\prime \prime}\right) & \text { if } i \neq j .\end{cases}
$$

It can be shown that a function of the form $\beta x^{n} \exp \left(-\alpha x^{2}\right)$ is bounded for any fixed $\beta, \alpha>0$, and $n \in \mathbb{N}$. The SE kernel (20) and all the above functions can be written as sums and products of functions of this form. Therefore, they are all bounded.

Lemma 3: Given Assumption 1, for any vector s, the following functions are bounded

1) $\partial \boldsymbol{\Sigma}(\mathbf{s}) / \partial s_{j}$.

2) $\partial^{2} \boldsymbol{\Sigma}(\mathbf{s}) / \partial s_{i} \partial s_{j}$.

Proof: Given the explicit form of $\partial \boldsymbol{\Sigma}(\mathbf{s}) / \partial s_{j}$ in (19), $\partial \mathbf{K}_{\mathcal{I}_{t}, \mathcal{I}_{t}}(\mathbf{s}) / \partial s_{j}, \partial \mathbf{K}_{\mathcal{I}_{t}}(\mathbf{s}) / \partial s_{j}$ and $\mathbf{K}_{\mathcal{I}_{t}}(\mathbf{s})$ are bounded as a result of Lemma 2. Moreover, from Lemma $1,\left(\mathbf{K}+\sigma_{n}^{2} \mathbb{I}\right)^{-1}$ is bounded, which leads to boundedness of $\partial \boldsymbol{\Sigma}(\mathbf{s}) / \partial s_{j}$.

The explicit form of $\partial^{2} \mathbf{\Sigma}(\mathbf{s}) / \partial s_{i} \partial s_{j}$ can be derived as

$$
\begin{array}{r}
\frac{\partial^{2} \mathbf{\Sigma}(\mathbf{s})}{\partial s_{i} \partial s_{j}}=\frac{\partial^{2} \mathbf{K}_{\mathcal{I}_{t}, \mathcal{I}_{t}}}{\partial s_{i} \partial s_{j}}-2 \mathbf{K}_{\mathcal{I}_{t}}\left(\mathbf{K}+\sigma_{n}^{2} \mathbb{I}\right)^{-1}\left(\frac{\partial^{2} \mathbf{K}_{\mathcal{I}_{t}}}{\partial s_{i} \partial s_{j}}\right)^{T} \\
-2\left(\frac{\partial \mathbf{K}_{\mathcal{I}_{t}}}{\partial s_{i}}\right)\left(\mathbf{K}+\sigma_{n}^{2} \mathbb{I}\right)^{-1}\left(\frac{\partial \mathbf{K}_{\mathcal{I}_{t}}}{\partial s_{j}}\right)^{T} .
\end{array}
$$

Given Lemma 2, it is straightforward to show that $\partial^{2} \boldsymbol{\Sigma}(\mathbf{s}) / \partial s_{i} \partial s_{j}$ is bounded.

Lemma 4: The function $f(\cdot)$ has a Lipschitz continuous gradient with a Lipschitz constant $L$.

Proof: We have $\nabla f(\mathbf{x})=\left[\nabla_{\mathbf{s}}^{T} f(\mathbf{x}), \nabla_{\mathbf{c}}^{T} f(\mathbf{x})\right]^{T}=$ $\left[\nabla^{T} \mathcal{H}(\mathbf{s}), \mathbf{0}^{T}\right]^{T}$, where $\nabla_{\mathbf{s}} f(\mathbf{x})$ and $\nabla_{\mathbf{c}} f(\mathbf{x})$ denote the gradient of $f(\mathbf{x})$ with respect to $\mathbf{s}$ and $\mathbf{c}$, respectively. Hence, we need to prove that $\nabla^{T} \mathcal{H}(\mathbf{s})$ is Lipschitz continuous, or equivalently, the Hessian matrix $\nabla^{2} \mathcal{H}(\mathbf{s})$ is bounded. From (18), each entry of the Hessian matrix is given as follows.

$$
\begin{aligned}
& \frac{\partial^{2} \mathcal{H}(\mathbf{s})}{\partial s_{i} \partial s_{j}}=-\operatorname{tr}\left(\left(\boldsymbol{\Sigma}(\mathbf{s})+\epsilon^{2} \mathbb{I}\right)^{-1} \frac{\partial^{2} \boldsymbol{\Sigma}(\mathbf{s})}{\partial s_{i} \partial s_{j}}\right. \\
& \left.-\left(\boldsymbol{\Sigma}(\mathbf{s})+\epsilon^{2} \mathbb{I}\right)^{-1} \frac{\partial \boldsymbol{\Sigma}(\mathbf{s})}{\partial s_{i}}\left(\boldsymbol{\Sigma}(\mathbf{s})+\epsilon^{2} \mathbb{I}\right)^{-1} \frac{\partial \boldsymbol{\Sigma}(\mathbf{s})}{\partial s_{j}}\right) .
\end{aligned}
$$

Given the results obtained in Lemmas 1 and $3,\left(\boldsymbol{\Sigma}(\mathbf{s})+\epsilon^{2} \mathbb{I}\right)^{-1}$, $\partial \boldsymbol{\Sigma}(\mathbf{s}) / \partial s_{i}, \partial \boldsymbol{\Sigma}(\mathbf{s}) / \partial s_{j}$ and $\partial^{2} \boldsymbol{\Sigma}(\mathbf{s}) / \partial s_{i} \partial s_{j}$ are bounded, which yield $\nabla^{2} \mathcal{H}(\cdot)$ is bounded from (22). Therefore, the Hessian matrix $\nabla^{2} f(\cdot)$ of $f(\cdot)$ is bounded. Furthermore, as $\nabla f(\cdot)$ is continuous and differentiable, $\nabla f(\cdot)$ is Lipschitz continuous according to the mean value theorem. The proof is complete.

Lemma 5: The function $I_{\mathcal{C}}(\cdot)$ is lower semi-continuous.

Proof: As $\mathcal{C}$ is the intersection of a closed discrete set, the compact set $\mathcal{Q}$ and some halfspaces, $\mathcal{C}$ is a closed set. Therefore, $I_{\mathcal{C}}(\cdot)$ is lower semi-continuous since it is the indicator function of a closed set.

Given Lemmas 4 and 5, we are now able to derive a convergence property for the proposed algorithm if the following assumption on the parameter of the proximal ADMM holds.

Assumption 2: The parameter $\rho$ is chosen such that $\rho>5 L$.

Theorem 1: Given Assumptions 1 and 2, if the sequence $\left\{\left(\mathbf{x}^{(k+1)}, \mathbf{z}^{(k+1)}, \mathbf{u}^{(k+1)}\right)\right\}$ generated by Algorithm 1 has a cluster point ${ }^{1}\left\{\left(\mathbf{x}^{*}, \mathbf{z}^{*}, \mathbf{u}^{*}\right)\right\}$, then Algorithm 1 converges, i.e.,

$$
\begin{aligned}
\lim _{k \rightarrow \infty}\left\|\mathbf{x}^{(k+1)}-\mathbf{x}^{(k)}\right\|^{2} & +\left\|\mathbf{z}^{(k+1)}-\mathbf{z}^{(k)}\right\|^{2} \\
& +\left\|\mathbf{u}^{(k+1)}-\mathbf{u}^{(k)}\right\|^{2}=0 .
\end{aligned}
$$

That is, any cluster point of the sequence is a stationary point for (13), i.e.,

$$
\nabla f\left(\mathbf{x}^{*}\right)=\mathbf{u}^{*}, \mathbf{u}^{*} \in \partial I_{\mathcal{C}}\left(\mathbf{z}^{*}\right), \mathbf{x}^{*}=\mathbf{z}^{*} .
$$

Proof: This result is proved by applying Lemmas 4 and 5 and using Theorem 1 in [12].

Note that the obtained stationary point is not globally unique unless the augmented Lagrangian $\mathcal{L}(14)$ is a KurdykaLojasiewicz (KL) function [12].

\section{Simulation Results and Discussions}

We verified the effectiveness of the proposed approach by extensive simulations in a synthetic environment using the real-world data set in [41] from the Intel Berkeley Lab. 


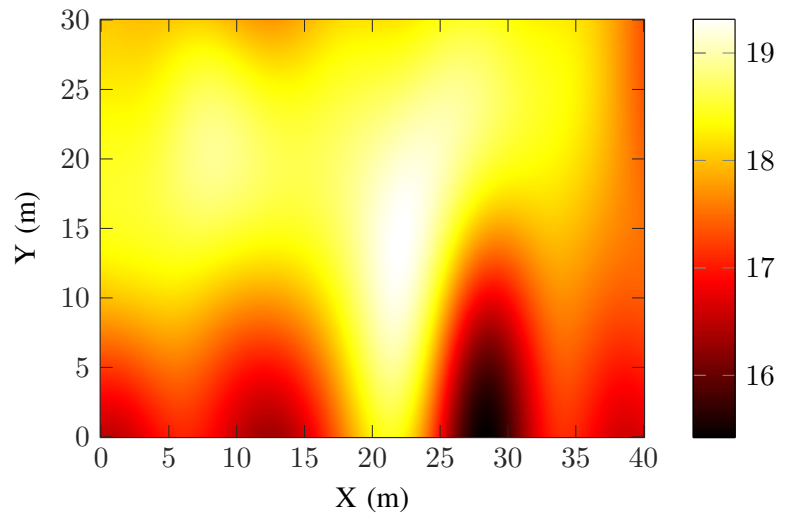

Fig. 2: A generated ground-truth of the temperature field.

\section{A. Simulation Setup}

The simulations were executed on a DELL computer with a $3.0 \mathrm{GHz}$ Intel Core i5 CPU and $8 \mathrm{~Gb}$ RAM. The algorithms were implemented in the Julia programming language. The JuMP package [42] and the Gurobi optimizer [43] were used to construct and solve the MIQP problems. In order to generate sensor measurements and evaluate the model accuracy, a simulated environmental phenomenon was created based on the real data set [41]. Specifically, a ground truth GP model was learned from 54 indoor temperature observations collected by 54 sensors deployed in the Intel Berkeley Research Lab at a specific time instant. The ground truth GP was then used to generate the 'virtual' measurements where the mobile sensors took measurements and the spatial field data where the learned GP models were assessed. The environmental field for the entire region of size $40 \mathrm{~m}$-by- $30 \mathrm{~m}$ generated by the ground truth GP is depicted in Figure 2.

In the simulations, a network of 5 robotic sensors was employed to monitor the temperature phenomenon in the region of interest: $\mathcal{Q}:=\{(x, y) \mid 0 \mathrm{~m} \leq x \leq 40 \mathrm{~m}, 0 \mathrm{~m} \leq$ $y \leq 30 \mathrm{~m}\}$. The bounds on the distance travelled by each robot between two consecutive sampling steps were set as $\Delta \mathbf{s}_{\min }=[-2,-2]^{T}(\mathrm{~m})$ and $\Delta \mathbf{s}_{\max }=[2,2]^{T}(\mathrm{~m})$. The parameters of the proximal ADMM algorithm were selected as $\rho=1, L=0.1, \epsilon_{\mathrm{res}}=10^{-3}$, and $k_{\max }=100$. In each simulation, the mobile sensors were set to conduct 15 sampling steps; that is, a total of 80 measurements were gathered by the network at the end of the data collection. Two stationary obstacles were positioned in the environment as represented by the gray rectangles in Fig. 4. Since in practice the mobile robots can start from any random initial locations in the environment, their starting locations in each simulation were chosen randomly but must be feasible in the environment. For a comprehensive evaluation of our proposed approach, we conducted 1000 experiments. Three prediction horizons of $H=1$ (for the single-step prediction based method), $H=3$ and $H=5$ (for the multi-step predictions based algorithm) were considered in the validation process.

\footnotetext{
${ }^{1}$ A point $x^{*}$ is a cluster point of a sequence $\left\{x_{n}\right\}$ if and only if there is a subsequence $\left\{x_{n_{k}}\right\}$ converging to $x^{*}$.
}

\section{B. Results and Discussions}

The convergence properties of the proximal ADMM algorithm in the first time step of a specific simulation are illustrated in Figures $3 a, 3 b$ and $3 c$ for the norm of the residuals $\left\|\epsilon^{(k)}\right\|=\left\|\mathbf{z}^{(k)}-\mathbf{x}^{(k)}\right\|$, the objective value $f_{0}\left(\mathbf{x}^{(k)}\right)$, and the first element of the dual variable $\mathbf{u}^{(k)}$, respectively. The results show that the proposed algorithm can achieve convergence of the residuals, objective, and dual variables.

To demonstrate and compare the performance of the singlestep and the multi-step approaches, we summarize the results of a particular simulation in Figures 4 and 5. For instance, in this experiment, the full 15-step sampling paths of the mobile sensors for the single-step method with $H=1$ and the multistep method with $H=5$ are illustrated in Figures $4 \mathrm{c}$ and $4 \mathrm{f}$, respectively. Moreover, the temperature fields in the entire environment predicted by the collective sensor measurements up to time steps $t=5, t=10$, and $t=15$, obtained by the two methods, are depicted in Fig. 5. It can be seen that the more measurements are gathered, the more accurate the prediction results are compared with the ground truth (Fig. 2). The corresponding predictive variances are demonstrated as background in Fig. 4. It is noted that both the multi-step and single-step adaptive sampling strategies successfully drove the robotic sensors to explore areas associated with a higher level of uncertainty while preventing robot-to-robot and robotto-obstacle collisions. Nevertheless, because the multi-step approach could predict possible sensor measurements multiple sampling steps ahead, it drove the mobile sensors to navigate the environment more efficiently, leading to more accurate prediction results. Over time, the prediction uncertainties obtained by the multi-step strategy are reduced faster and more uniformly than those obtained by the single-step strategy, as can be seen in Fig. 4.

To further validate the benefits of using multi-step predictions in the proposed approach, we examined three different validation metrics for the predictions, including the maximum variances (MVs), the root mean squared errors (RMSEs), and the maximum absolute errors (MAEs) for three horizon lengths $H=1,3,5$. The results of an example simulation computed over 15 sampling steps are shown in Fig. 6, where the multistep approaches achieved faster and larger reductions of the prediction errors as compared with the single-step approach. It can be seen that once an adequate amount of measurements have been collected, e.g., after 11 time steps, the longer the prediction horizon $H$ is, the more accurate the prediction results are. Furthermore, we summarized the statistics of the three validation metrics at the $15^{\text {th }}$ time step (the final time step) for all 1000 simulations in the box plots in Fig. 7. It is obvious that the multi-step predictions in our proposed method led to considerably more accurate prediction results.

The statistics of the computation time of our algorithm in each time step over the 1000 simulations are summarized in Fig. 8 for different horizon lengths. Unsurprisingly, the computation time is proportional to the length of the prediction horizon. More specifically, on average, in each time step, the single-step approach took approximately $0.6 \mathrm{~s}$ while the multistep approaches with $H=3$ and $H=5$ took about $1.4 \mathrm{~s}$ 


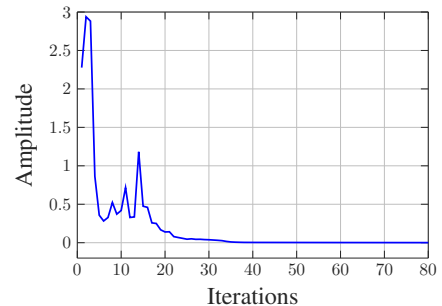

(a) Residual convergence

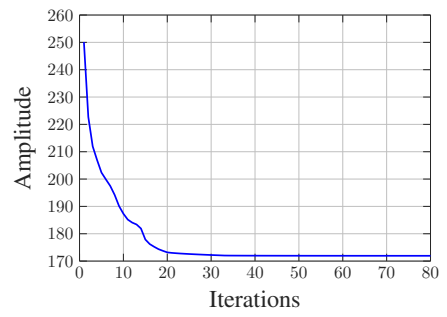

(b) Objective convergence

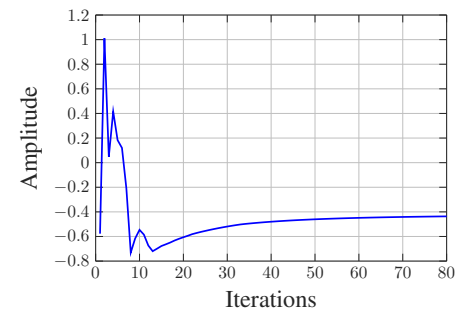

(c) Dual variable convergence

Fig. 3: Convergence properties of the proximal ADMM algorithm in a specific time step.

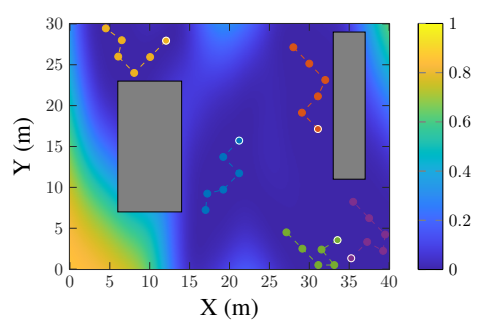

(a) Time step 5

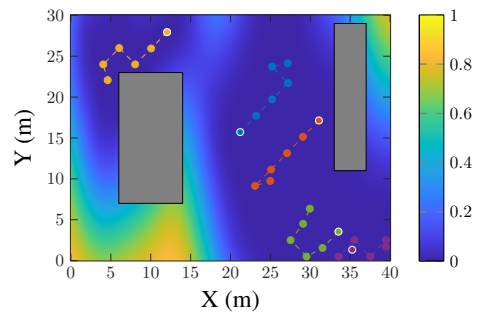

(d) Time step 5

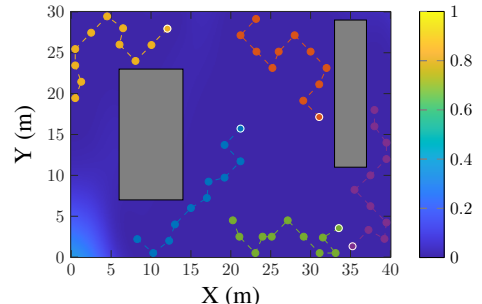

(b) Time step 10

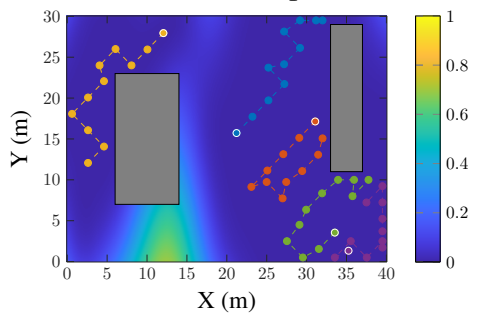

(e) Time step 10

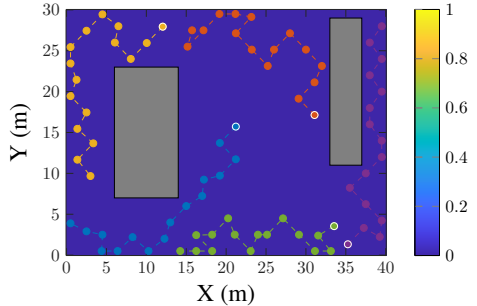

(c) Time step 15

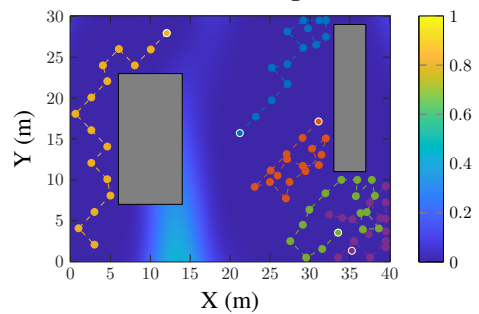

(f) Time step 15

Fig. 4: The predictive variances in the entire environment and the sampling trajectories of the mobile sensors obtained by the multi-step prediction approach $H=5$ (three top figures) versus the single-step prediction approach $H=1$ (three bottom figures) in a simulation with stationary obstacles (gray blocks). Filled dots are the sampling locations.

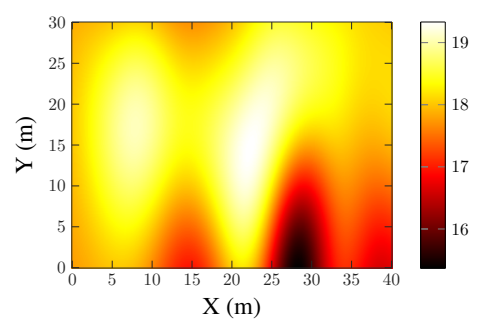

(a) Time step 5

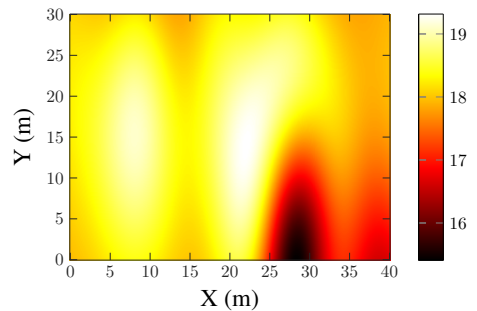

(d) Time step 5

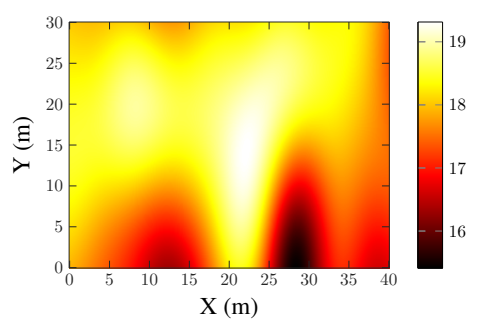

(b) Time step 10

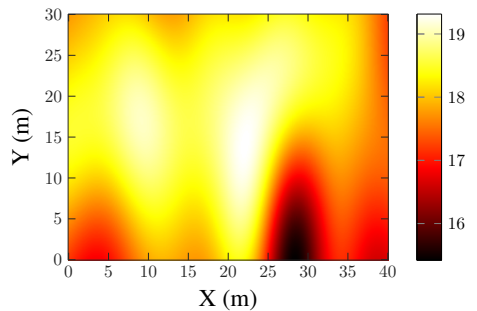

(e) Time step 10

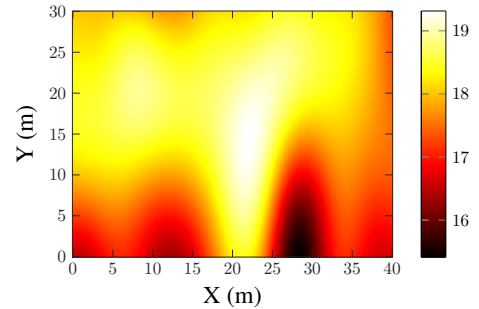

(c) Time step 15

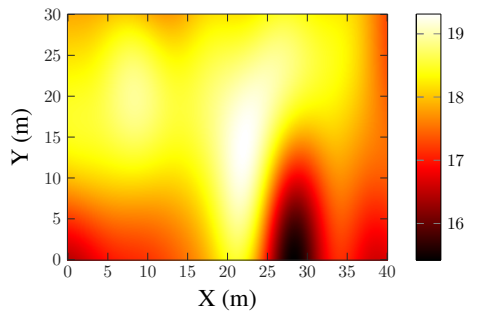

(f) Time step 15

Fig. 5: The predictive means in the entire environment obtained by the multi-step prediction approach $H=5$ (three top figures) versus the single-step prediction approach $H=1$ (three bottom figures) in a simulation with stationary obstacles.

and $2.0 \mathrm{~s}$, respectively. Therefore, in practice, depending on the application, the computational resources, and the timing requirements, the horizon length can be chosen appropriately to balance between the model accuracy and the computational 
burden.

\section{CONCLUSiOnS}

This paper has presented a novel efficient method for adaptive sampling in an MRSN monitoring spatial fields. By employing a GP model to represent the spatial phenomenon, possible sensor measurements can be predicted at multiple sampling steps ahead, allowing a receding horizon adaptive sampling optimization problem to be formulated to find the optimal multi-step sampling paths for the mobile sensors. Exploitation of the multi-step look-ahead predictions in the sampling strategy can provide a resource- and obstacleconstrained MRSN with significant benefits, including more efficient navigation and coordination among the mobile sensors, and more informative data collection. Though the mixedinteger optimization problem is non-convex and complex, it can be efficiently solved by the proximal ADMM algorithm proposed in this paper. Furthermore, if the common SE kernel is used for the GP model, it is formally proved that the solution obtained by the proposed algorithm can converge to a stationary point. An extensive evaluation conducted in a synthetic environment based on a real-world data set verified the efficacy of our proposed approach.

\section{REFERENCES}

[1] M. Bacco, F. Delmastro, E. Ferro, and A. Gotta, "Environmental monitoring for smart cities," IEEE Sensors Journal, vol. 17, no. 23, pp. 7767-7774, 2017.

[2] S. J. Ramson and D. J. Moni, "Applications of wireless sensor networks-a survey," in 2017 International Conference on Innovations in Electrical, Electronics, Instrumentation and Media Technology (ICEEIMT). IEEE, 2017, pp. 325-329.

[3] T. Arampatzis, J. Lygeros, and S. Manesis, "A survey of applications of wireless sensors and wireless sensor networks," in Proceedings of the 2005 IEEE International Symposium on, Mediterrean Conference on Control and Automation Intelligent Control, 2005. IEEE, 2005, pp. 719-724.

[4] H. Xiao, R. Cui, and D. Xu, "A sampling-based bayesian approach for cooperative multiagent online search with resource constraints," IEEE Transactions on Cybernetics, vol. 48, no. 6, pp. 1773-1785, 2017.

[5] C. K. Williams and C. E. Rasmussen, Gaussian processes for machine learning. MIT press Cambridge, MA, 2006, vol. 2, no. 3.

[6] A. E. Gelfand and E. M. Schliep, "Spatial statistics and Gaussian processes: A beautiful marriage," Spatial Statistics, vol. 18, pp. 86-104, 2016.

[7] A. Jain and E. Y. Chang, "Adaptive sampling for sensor networks," in Proceedings of the 1st International Workshop on Data Management for Sensor Networks: in conjunction with VLDB 2004, 2004, pp. 10-16.

[8] L. Nguyen, N. Ulapane, and J. V. Miro, "Adaptive sampling for spatial prediction in environmental monitoring using wireless sensor networks: A review," in 2018 13th IEEE Conference on Industrial Electronics and Applications (ICIEA). IEEE, 2018, pp. 346-351.

[9] D. A. Paley and A. Wolek, "Mobile Sensor Networks and Control: Adaptive Sampling of Spatiotemporal Processes," Annual Review of Control, Robotics, and Autonomous Systems, vol. 3, pp. 91-114, 2020, publisher: Annual Reviews.

[10] B. Alrifaee, M. G. Mamaghani, and D. Abel, "Centralized non-convex model predictive control for cooperative collision avoidance of networked vehicles," in 2014 IEEE International Symposium on Intelligent Control (ISIC). IEEE, 2014, pp. 1583-1588.

[11] V.-A. Le, L. Nguyen, and T. X. Nghiem, "An Efficient Adaptive Sampling Approach for Mobile Robotic Sensor Networks using Proximal ADMM," in 2021 American Control Conference (ACC). IEEE, 2021.

[12] G. Li and T. K. Pong, "Global convergence of splitting methods for nonconvex composite optimization," SIAM Journal on Optimization, vol. 25, no. 4, pp. 2434-2460, 2015.

[13] M. Hong, Z.-Q. Luo, and M. Razaviyayn, "Convergence analysis of alternating direction method of multipliers for a family of nonconvex problems," SIAM Journal on Optimization, vol. 26, no. 1, pp. 337-364, 2016, publisher: SIAM.
[14] J. Chen, K. H. Low, Y. Yao, and P. Jaillet, "Gaussian process decentralized data fusion and active sensing for spatiotemporal traffic modeling and prediction in mobility-on-demand systems," IEEE Transactions on Automation Science and Engineering, vol. 12, no. 3, pp. 901-921, 2015, publisher: IEEE.

[15] K. Tiwari, V. Honoré, S. Jeong, N. Y. Chong, and M. P. Deisenroth, "Resource-constrained decentralized active sensing for multi-robot systems using distributed Gaussian processes," in 2016 16th International Conference on Control, Automation and Systems (ICCAS). IEEE, 2016, pp. $13-18$

[16] S. Kemna, J. G. Rogers, C. Nieto-Granda, S. Young, and G. S. Sukhatme, "Multi-robot coordination through dynamic voronoi partitioning for informative adaptive sampling in communication-constrained environments," in 2017 IEEE International Conference on Robotics and Automation (ICRA). IEEE, 2017, pp. 2124-2130.

[17] H. M. La and W. Sheng, "Distributed sensor fusion for scalar field mapping using mobile sensor networks," IEEE Transactions on Cybernetics, vol. 43, no. 2, pp. 766-778, 2013.

[18] Q. Lu, Q.-L. Han, B. Zhang, D. Liu, and S. Liu, "Cooperative control of mobile sensor networks for environmental monitoring: An eventtriggered finite-time control scheme," IEEE Transactions on Cybernetics, vol. 47, no. 12 , pp. 4134-4147, 2016.

[19] A. Viseras, T. Wiedemann, C. Manss, L. Magel, J. Mueller, D. Shutin, and L. Merino, "Decentralized multi-agent exploration with onlinelearning of gaussian processes," in 2016 IEEE International Conference on Robotics and Automation (ICRA). IEEE, 2016, pp. 4222-4229.

[20] Y. Xu, J. Choi, S. Dass, and T. Maiti, "Sequential Bayesian prediction and adaptive sampling algorithms for mobile sensor networks," IEEE Transactions on Automatic Control, vol. 57, no. 8, pp. 2078-2084, 2011, publisher: IEEE.

[21] Y. Xu, J. Choi, and S. Oh, "Mobile sensor network navigation using gaussian processes with truncated observations," IEEE Transactions on Robotics, vol. 27, no. 6, pp. 1118-1131, 2011, publisher: IEEE.

[22] Y. Xu, J. Choi, S. Dass, and T. Maiti, "Efficient Bayesian spatial prediction with mobile sensor networks using Gaussian Markov random fields," Automatica, vol. 49, no. 12, pp. 3520-3530, 2013, publisher: Elsevier.

[23] R. Ouyang, K. H. Low, J. Chen, and P. Jaillet, "Multi-robot active sensing of non-stationary gaussian process-based environmental phenomena," in Proceedings of the 2014 International Conference on Autonomous Agents and Multiagent Systems, 2014, pp. 573-580.

[24] R. Marchant and F. Ramos, "Bayesian optimisation for intelligent environmental monitoring," in 2012 IEEE/RSJ International Conference on Intelligent Robots and Systems. IEEE, 2012, pp. 2242-2249.

[25] Q. Lu and Q.-L. Han, "Mobile robot networks for environmental monitoring: A cooperative receding horizon temporal logic control approach," IEEE Transactions on Cybernetics, vol. 49, no. 2, pp. 698-711, 2018.

[26] Z. Wang, H.-X. Li, and C. Chen, "Reinforcement learning-based optimal sensor placement for spatiotemporal modeling," IEEE Transactions on Cybernetics, vol. 50, no. 6, pp. 2861-2871, 2019

[27] D. Jang, J. Yoo, C. Y. Son, D. Kim, and H. J. Kim, "Multi-robot active sensing and environmental model learning with distributed Gaussian process," IEEE Robotics and Automation Letters, vol. 5, no. 4, pp. 59055912, 2020.

[28] W. Luo and K. Sycara, "Adaptive sampling and online learning in multirobot sensor coverage with mixture of gaussian processes," in 2018 IEEE International Conference on Robotics and Automation (ICRA). IEEE, 2018, pp. 6359-6364.

[29] W. Luo, C. Nam, G. Kantor, and K. Sycara, "Distributed environmental modeling and adaptive sampling for multi-robot sensor coverage," in Proceedings of the 18th International Conference on Autonomous Agents and Multiagent Systems, 2019, pp. 1488-1496.

[30] Y. Xu and J. Choi, "Adaptive sampling for learning Gaussian processes using mobile sensor networks," Sensors, vol. 11, no. 3, pp. 3051-3066, 2011, publisher: Molecular Diversity Preservation International.

[31] A. Krause, A. Singh, and C. Guestrin, "Near-optimal sensor placements in gaussian processes: Theory, efficient algorithms and empirical studies." Journal of Machine Learning Research, vol. 9, no. 2, 2008.

[32] T. Li, K. Tong, M. Xia, B. Li, and C. W. de Silva, "Information-based hierarchical planning for a mobile sensing network in environmental mapping," IEEE Systems Journal, vol. 14, no. 2, pp. 1692-1703, 2019.

[33] Y. T. Tan, A. Kunapareddy, and M. Kobilarov, "Gaussian process adaptive sampling using the cross-entropy method for environmental sensing and monitoring," in 2018 IEEE International Conference on Robotics and Automation (ICRA). IEEE, 2018, pp. 6220-6227. 


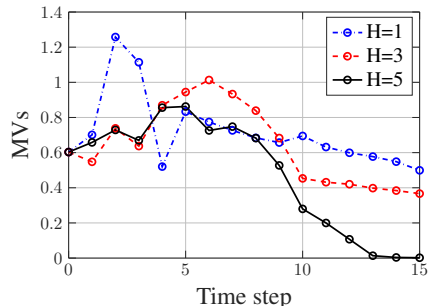

(a) The maximum variances

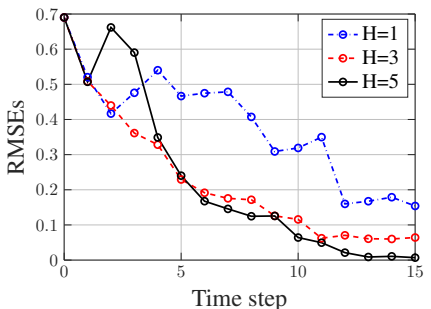

(b) The root mean squared errors

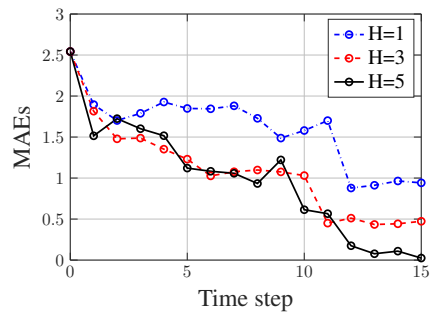

(c) The maximum absolute errors

Fig. 6: The validation metrics computed over time in one simulation.

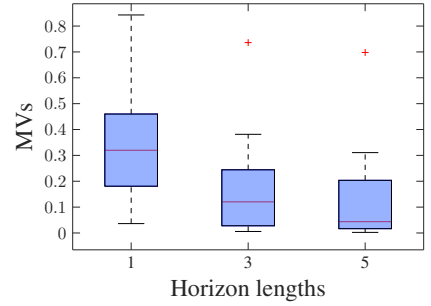

(a) The maximum variances

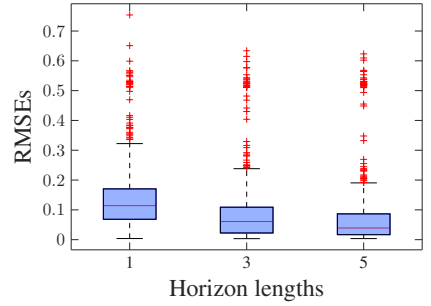

(b) The root mean squared errors

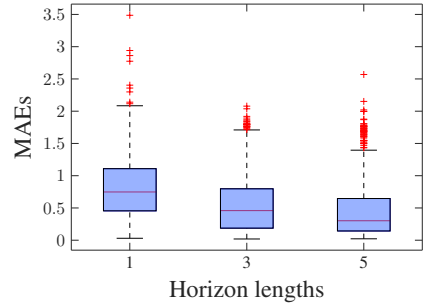

(c) The maximum absolute errors

Fig. 7: The validation metrics computed at the $15^{\text {th }}$ time step, summarized for 1000 simulations.

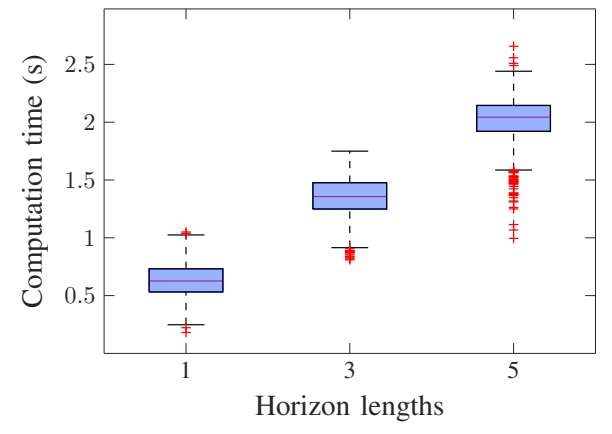

Fig. 8: Statistics of the computation time for 1000 simulations.

[34] L. V. Nguyen, S. Kodagoda, and R. Ranasinghe, "Spatial sensor selection via Gaussian Markov random fields," IEEE Transactions on Systems, Man, and Cybernetics: Systems, vol. 46, no. 9, pp. 1226-1239, 2015.

[35] L. Nguyen, S. Kodagoda, R. Ranasinghe, and G. Dissanayake, "Mobile robotic sensors for environmental monitoring using Gaussian Markov random field," Robotica, vol. 39, no. 5, pp. 862-884, 2021.

[36] L. V. Nguyen, S. Kodagoda, R. Ranasinghe, and G. Dissanayake, "Information-driven adaptive sampling strategy for mobile robotic wireless sensor network," IEEE Transactions on Control Systems Technology, vol. 24, no. 1, pp. 372-379, 2015, publisher: IEEE.

[37] _ "Adaptive placement for mobile sensors in spatial prediction under locational errors," IEEE Sensors Journal, vol. 17, no. 3, pp. 794-802, 2016, publisher: IEEE.

[38] V.-A. Le, L. Nguyen, and T. X. Nghiem, "ADMM-based Adaptive Sampling Strategy for Nonholonomic Mobile Robotic Sensor Networks," IEEE Sensors Journal, 2021.

[39] T. M. Cover and J. A. Thomas, "Information theory and statistics," Elements of Information Theory, vol. 1, no. 1, pp. 279-335, 1991.

[40] Y. Wang, W. Yin, and J. Zeng, "Global convergence of ADMM in nonconvex nonsmooth optimization," Journal of Scientific Computing, vol. 78, no. 1, pp. 29-63, 2019.

[41] "Intel Lab Data," 2004. [Online]. Available: http://db.csail.mit.edu/labdata/labdata.html

[42] I. Dunning, J. Huchette, and M. Lubin, "JuMP: A modeling language for mathematical optimization," SIAM review, vol. 59, no. 2, pp. 295-320, 2017.

[43] Gurobi Optimization, LLC, "Gurobi optimizer reference manual," 2021. [Online]. Available: http://www.gurobi.com 